\title{
Artificial Bee Colony Algorithm for Image Restoration
}

\author{
Jie Yang, Dan Hu and Yu Weiyu \\ School of Electronic and Information Engineering, South China University of Technology, \\ Guangzhou, P. R. China
}

email: 2368419792@qq.com,254232612@qq.com,yuweiyu@scut.edu.cn

Keywords: ABC algorithm; Wiener Filter; Image Restoration

\begin{abstract}
This paper proposes a new method for image restoration using artificial bee colony algorithm (ABC algorithm). ABC algorithm combined with wiener filter is used to restore image. In the fuzzy identification, ABC algorithm is used for optimization the point spread function (PSF). In the restoring part, wiener filter is used for image restoration. Proposed method overcomes the problem of the traditional image restoring, which needs to know the PSF in advance. In addition, this method improves the performance of the traditional ABC algorithm by initializing the first group of bee colony. The pretreatment can reduce the randomness and improve the performance of ABC algorithm. Experimental results showed that the proposed method is effective for image restoration.
\end{abstract}

\section{Introduction}

Image restoration is the basis of many fields of research such as image processing, computer vision and so on. In the process of acquiring an image, the quality of the image often decreases because of various reasons such as motion blur, noise, and camera miscues. The process of linear image degradation is usually like as following model:

$$
g=h * f+n
$$

Here, $g$ represents blurred image. $h$ represents the point spread function. $f$ represents the original image and $n$ represents the noise. The purpose of image restoration is to "compensate for" or "undo" defects which degrade an image. In the traditional process of image restoration, the PSF of blurred image needs to be known in advance to restore image. However, in many practical situations, the PSF is generally difficult to determine. The information of degradation must be extracted from the blurred image to restore image. This process is called blind image restoration [1][2].

Blind image restoration is a more difficult image restoration method where image recovery is performed with little or no prior knowledge of the degrading PSF. Methods for blind image restoration can be divided into two categories. One is to identify the PSF and then estimate the image by image restoring algorithm. The other is the simultaneous estimation of the PSF and the original image[3]. In this paper, $A B C$ algorithm is used for obtaining the PSF in the fuzzy identification and wiener filter is used for image restoration in the restoring identification [4]. Fuzzy identification and restoring identification will be alternately iterated until obtaining the restored image.

ABC algorithm has many advantages such as convergence speed, strong robustness because of the information sharing and interaction of the bees. It can prevent premature entry into local optimum and improve the quality of the optimal solution. Meanwhile this paper improves the performance of the traditional ABC algorithm, using pretreatment to initialize the first group of bee colony. The pretreatment can reduce the randomness and improve the performance of $\mathrm{ABC}$ algorithm.

\section{Artificial bee colony (ABC) algorithm}

Artificial bee colony algorithm was first introduced by Dervis Karaboga and Basturk in 2005 for solving numerical optimization problems [5]. ABC algorithm has achieved better results on the optimization problems [6-9]. The bee colony consists of three kinds of bees: employed bees, 
onlookers and scouts [10]. Employed bees go to the food sources and come back to hive and dance on this area. Onlookers watch the dances of employed bees and choose food sources depending on dances. The employed bees whose food source has been abandoned become a scout and start to search for a new food source randomly. In ABC algorithm, the position of a food source represents a possible solution of the optimization problem and the nectar amount of a food source corresponds to the fitness of the associated solution. The steps of the algorithm as follows:

a) Initialize the population of bee colony. Set all the bees as the scouts which search nectar randomly.

b) Assess the effectiveness of each nectar according to the objective function $f$. Depending on the value of the objective function, the first half of the swarm consists of employed bees, and the second half consists of onlooker bees.

c) Each employed bee searches for new nectar in their respective areas in accordance with Equ. (2).

$$
\mathrm{V}_{i j}=x_{i j}+R_{i j}\left(x_{i j}-x_{k j}\right)
$$

Here, $V_{i j}$ represents the new location of nectar. ${ }^{x_{i j}}$ represents the j-dimensional position of the i-th nectar. ${ }^{x_{k j}}$ represents the j-dimensional position of the k-th $\operatorname{nectar}\left({ }^{k} \neq i\right) \cdot R_{i j}$ is a random number between -1 to 1 .

d) Determine whether to update the original nectar based on greedy selection strategy.

e) An onlooker bee evaluates the nectar information taken from all employed bees and chooses a food source with a probability related to its nectar amount. This probabilistic selection is really a roulette wheel selection mechanism which is described as Equ.(3):

$$
P_{i}=\text { fit }_{j} / \sum_{j} \text { fit }_{j}
$$

Where fit ${ }_{i \mathrm{i}}$ represents the fitness value of the $e^{i^{\text {th }}}$ solution in the swarm. As seen, the better the solution i, the higher the probability of the ${ }^{i^{\text {th }}}$ food source selected. Each onlooker search for new nectar in the selected areas and determine whether to update the original nectar based on greedy selection strategy.

f) If a position cannot be improved over a predefined number of cycles, then the food source is abandoned. Assume that the abandoned source is $\mathrm{X}_{\mathrm{i}}$, and then the scout bee discovers a new food source to be replaced with ${ }^{i^{t h}}$ as below:

$$
X_{i k}=1 b_{j}+\operatorname{rand}(0,1) \times\left(u b_{j}-1 b_{j}\right)
$$

Where $\operatorname{rand}(0,1)$ is a random number within $[0,1]$ based on a normal distribution. $1 b, u b$ are lower and upper boundaries of the $i_{t h}$ dimension respectively.

g) Record the optional solution at this iteration. Repeat the above steps until the end of the cycle. End algorithm.

In ABC algorithm, employed bees have to maintain a good nectar source. Onlookers are helpful to improve the effects of the convergence rate. Scouts can help the algorithm not to be trapped in the local minima. The termination condition of the algorithm loop can be set to the number of cycles down to zero.

\section{Wiener filter}

Wiener filter was proposed by Wiener firstly and it achieved better performance in imagel processing, especially in image restoration [11-13].

The objective of wiener filter is to find the estimates to make a statistical error function to be minimized as Equ.(5):

$$
e^{2}=E\left\{(f-\hat{f})^{2}\right\}
$$

$E$ is the expected value. $f$ is the original image and it can also be expressed in the frequency domain as Equ.(6): 


$$
F\left(u, v=\frac{1}{H(u, v)} \frac{\wedge}{\mid(u, v)^{2}+S_{f}(u, v) / S_{\eta}(u, v)}\right) G(u, v)
$$

Here, $H(u, v)$ represents the degradation function. It is the fourier transform of the point spread function.

$|H(u, v)|^{2}=H *(u, v) H(u, v)$

$H *(u, v)$ represents the complex conjugate of $H(u, v)$.

$S_{\eta}(u, v)=|N(u, v)|^{2}$ represents the power spectrum of noise.

$S_{f}(u, v)=|F(u, v)|^{2}$ represents the power spectrum of the original image.

$S_{f} / S_{\eta}$ is called the signal to noise power ratio.

\section{Artificial Bee Colony Algorithm for Image Restoration}

Wiener filter makes the mean square error between original image and the recovery image minimum. Wiener filter needs to know the point spread function in advance for image restoration. However, in many case, the point spread function is unknown, so we can not use Wiener filter to restore the blurred image directly.

$\mathrm{ABC}$ algorithm is used to estimate the point spread function and then uses Wiener filter for image restoration.

$h(x, y)$ is estimated with the common traditional model of image restoration.

From Equ.(1),we know that:

$n=g-h * f$

Without the prior knowledge of $n$, the method of image restoration needs to find an estimate of $\mathrm{f}$, which makes $h * f$ closest to $\mathrm{g}$ in the sense of minimum mean square error. Therefore, image restoration can be seen to get the minimum as follows for $f$.

$$
\min (L(\hat{f}))=\min \left(\|g-h * f\|^{2}\right)
$$

$\wedge$ Similarly, h can be estimated according to the traditional model of image restoration. Initialize $f_{0}=g$ and estimate the $h$ which makes the formula as (9) minimum. Then use Wiener filter to restore the image with the estimated $h$ and finally obtain the restored image $f$. The algorithm is as follows:

a) Initialize $f=g$ ( $g$ is the blurred image) and the number of bees $s=50$. Each bee $h_{x}$ is set as the initial value of the point spread function.

Here, set $h_{x}$ as the array of $1 \times n$ and $N=M^{2}$ ( $\mathrm{M}$ is the dimension of the point spread function, and $\mathrm{M}$ is a positive integer). To reduce the randomness of the initial value of $\mathrm{ABC}$ algorithm, set $h_{1}-h_{10}$ as different random templates of Gaussian, $h_{11}-h_{20}$ as different random templates of Mean, $h_{21}-h_{30}$ as different random templates of Median, $h_{31}-h_{50}$ as different random templates.

b) The benefit function of $\mathrm{ABC}$ algorithm is shown in Equ(8)

Obtain the optimal solution $h_{\text {bestn }}$ which makes the benefit function minimum according to ABC algorithm and constraints $h_{\text {bestn }}$ as follows Equ(9):

$$
h_{\text {bestn }}(x, y)=\left\{\begin{array}{l}
h_{\text {bestn }}(x, y), h_{\text {bestn }}(x, y)>0 \\
0, \quad \text { others }
\end{array}\right.
$$


c) Then use Wiener filter to restore the image with the estimated of the point spread function $h_{\text {bestn }}$ and finally obtain the restored image $\hat{f}_{n}$.

d) With the $\hat{F}_{N}$, use $\mathrm{ABC}$ algorithm to get the new optimal solution $h_{b e s t(n+1)}$ by the Equ. (6) and get the next restored image $\hat{f}_{n+1}$ with the new estimated $h_{b e s t(n+1)}$.

Determine whether the termination condition is satisfied.Here, we set the number of iterations as the termination condition. If the termination condition is satisfied, the algorithm will be stopped. $f=\hat{f}_{n+1}$ is the final result of image restoration. Otherwise, set $f=\hat{f}_{n+1}$ and repeat the steps from b) to e).

\section{Experimental Results}

In order to verify the effectiveness of the proposed algorithm, we tested a number of images. It is assumed that a blurred image is produced by linear degradation model which is $g=h \times f$. The model of $h$ is unknown. The original images are shown in figure 1. The blurred images are shown in figure 2.
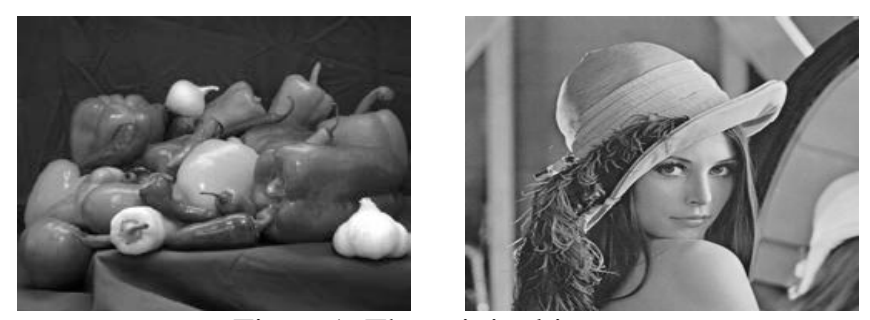

Figure1. The original image
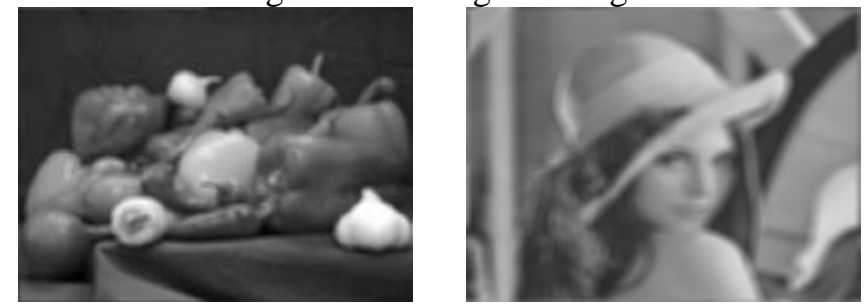

Figure2. the blurred image

In the experiment, the proposed method was used for image restoration. Depending on different dimensions of the PSF, the results of the experiment were as follows. Here, $\mathrm{M}$ represents the dimension of the PSF. For example, PSF represents the matrix of $3 \times 3$ when $\mathrm{M}=3$.

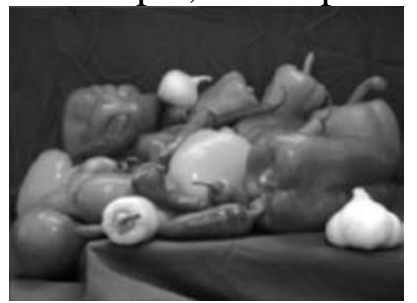

(a)

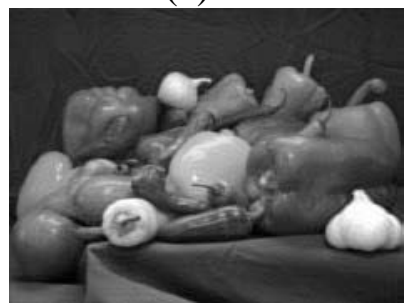

(c)

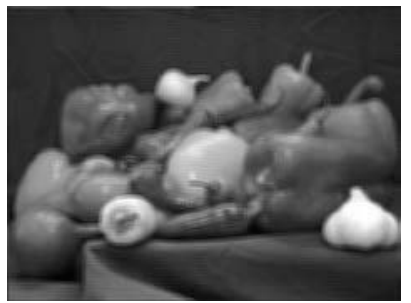

(b)

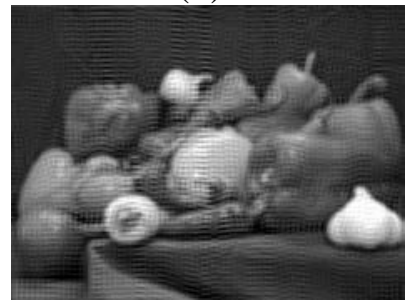

(d) 


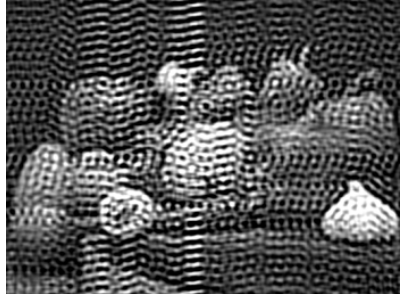

(e)

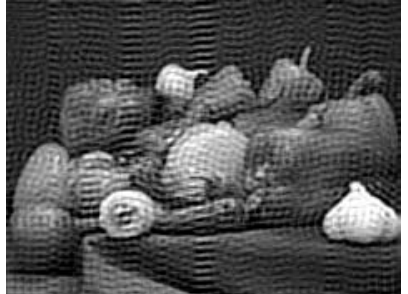

(f)
Figure3.(a) the result by $M=3 \quad$ (b) the result by $M=5$

(d) the result by $\mathrm{M}=9$

(e) the result by $M=11$

(c) the result by $\mathrm{M}=7$

(f) the result by $M=13$

Table1. the different results depending on different dimension $\mathbf{M}$

\begin{tabular}{lccccccc}
\hline & The blurred image & $\mathrm{M}=3$ & $\mathrm{M}=5$ & $\mathrm{M}=7$ & $\mathrm{M}=9$ & $\mathrm{M}=11$ & $\mathrm{M}=13$ \\
\hline value of PSNR & 28.932 & 29.023 & 28.217 & 26.101 & 33.985 & 22.328 & 14.351 \\
\hline
\end{tabular}

As can be seen from the above results of the experiment, the proposed method can effectively be applied to the image restoration. Different dimensions lead to different results.

In Fig4, when $M=9$, the effect of image restoration is the best. According to the analysis of peak signal to noise ratio(PSNR), the quality of restored image is worse than the original blurred image when $M=5,7,11,13$. When $M=9$, the PSNR is maximized and the effect of image restoration is best. In addition, when $\mathrm{M}=9$, the $\mathrm{ABC}$ algorithm and the particle swarm optimization (PSO)[14] were used on image restoration. The results of two different methods are shown as follows:

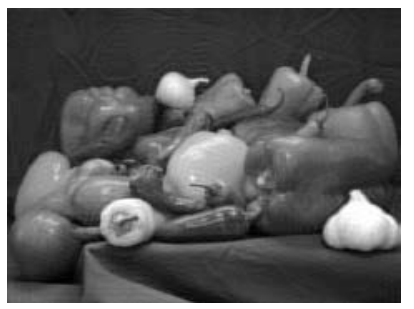

(a)-1

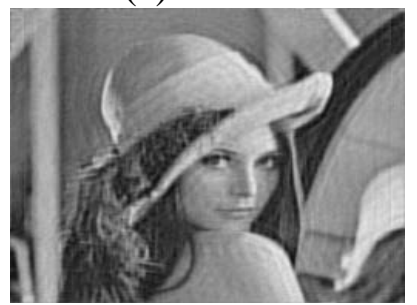

(b)-1

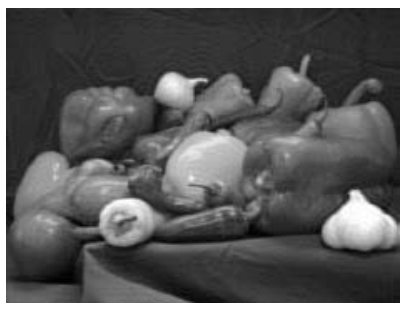

(a)-2

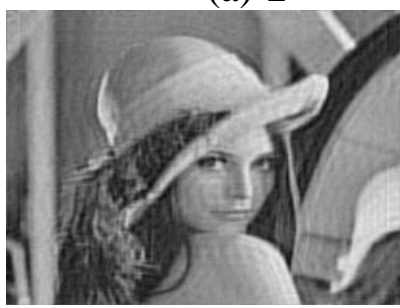

(b) -2

Figure4. (a)The result of different images based on the PSO

(b)The result of different images based on the ABC

Table2. the PSNR of image restoration by ABC and PSO

\begin{tabular}{cccc}
\hline The value of PSNR & The blurred image & PSO & ABC \\
\hline peppers & 28.93183 & 33.8601 & 33.9849 \\
\hline lena & 23.0214 & 27.8499 & 27.9623 \\
\hline
\end{tabular}

From the experimental results, both PSO and ABC can be effectively used for image restoration. As can be seen from Fig5, the results of the restored images by these two methods are about the same. According to the value of PSNR, the value of the image by ABC algorithm is a little higher than that by PSO. It means that the effect of the image by ABC algorithm is better than that by PSO. Experimental results show that the proposed method is effective for image restoration and it can be used to get a better recovery result.

\section{Conclusion}

In this paper, $\mathrm{ABC}$ algorithm combined with wiener filtering is used to restore image. In the 
fuzzy identification phase, ABC algorithm is used for obtaining the point spread function (PSF) and in the restoring phase, wiener filtering is used for image restoration. The experimental results show that the proposed method is effective for image restoration. With the proposed method, PSF does not have to be available in advance. This method improves the performance of the traditional ABC algorithm by initializing the first group. The pretreatment can reduce the randomness and improve the performance of ABC algorithm. The experimental results show that the proposed method is effective for image restoration.

\section{References}

[1] Liu J, Huang T Z, Lv X G, et al. Restoration of blurred color images with impulse noise[J]. Computers \& Mathematics with Applications, 2015, 70(6): 1255-1265.

[2] Zeng F, Wang W, Tang $M$, et al. Exposing Blurred Image Forgeries through Blind Image Restoration[C]//2015 10th International Conference on P2P, Parallel, Grid, Cloud and Internet Computing (3PGCIC). IEEE, 2015: 466-469.

[3] Dong C, Xie M. A blind image restoration algorithm based on nonlocal means and EM algorithm[C]//Audio, Language and Image Processing (ICALIP), 2012 International Conference on. IEEE, 2012: 485-489.

[4] Pham T D. Estimating parameters of optimal average and adaptive Wiener filters for image restoration with sEqu.ential Gaussian simulation [J]. IEEE Signal Processing Letters, 2015, 22(11): 1950-1954.

[5] Karaboga D, Basturk B. A powerful and efficient algorithm for numerical function optimization: artificial bee colony (ABC) algorithm [J]. Journal of global optimization, 2007, 39(3): 459-471.

[6] Cao Y F, Xiao Y H, Yu W Y, et al. Multi-level threshold image segmentation based on PSNR using artificial bee colony algorithm[J]. Research Journal of Applied Sciences, Engineering and Technology, 2012, 4(2): 104-107.

[7] Draa A, Bouaziz A. An artificial bee colony algorithm for image contrast enhancement[J]. Swarm and Evolutionary computation, 2014, 16: 69-84.

[8] Dakshitha B A, Deekshitha V, Manikantan K. A novel Bi-level Artificial Bee Colony algorithm and its application to image segmentation[C]//2015 IEEE International Conference on Computational Intelligence and Computing Research (ICCIC). IEEE, 2015: 1-7.

[9] Agrawal V, Chandra S. Feature selection using Artificial Bee Colony algorithm for medical image classification[C]//Contemporary Computing (IC3), 2015 Eighth International Conference on.IEEE, 2015: 171-176.

[10] Anuar S, Selamat A, Sallehuddin R. A modified scout bee for artificial bee colony algorithm and its performance on optimization problems[J]. Journal of King Saud University-Computer and Information Sciences, 2016.

[11] Mbarki Z, Seddik H, Braiek E B. A rapid hybrid algorithm for image restoration combining parametric Wiener filtering and wave atom transform[J]. Journal of Visual Communication and Image Representation, 2016.

[12] Figen Z G, Saldi N. Blind image restoration by employing an optimized wiener filter[C]//2012 20th Signal Processing and Communications Applications Conference (SIU). IEEE, 2012: 1-4.

[13] Yoo J C, Ahn C W. Image restoration by blind-Wiener filter[J]. IET Image Processing, 2014, 8(12): 815-823.

[14] Akay B. A study on particle swarm optimization and artificial bee colony algorithms for multilevel thresholding[J]. Applied Soft Computing, 2013, 13(6): 3066-3091. 